\title{
PRE-PLAY COMMUNICATION AND CREDIBILITY: A TEST OF AUMANN'S CONJECTURE*
}

\author{
Gary Charness \\ Department of Economics and Business \\ Universitat Pompeu Fabra \\ Barcelona, Spain 08005 \\ e-mail: charness@upf.es
}

June, 1998

\begin{abstract}
The effectiveness of pre-play communication in achieving efficient outcomes has long been a subject of controversy. In some environments, cheap talk may help to achieve coordination. However, Aumann conjectures that, in a variant of the Stag Hunt game, a signal for efficient play is not self-enforcing and concludes that an "agreement to play [the efficient outcome] conveys no information about what the players will do." Harsanyi and Selten (1988) cite this example as an illustration of risk-dominance vs. payoffdominance. Farrell and Rabin (1996) agree with the logic, but suspect that cheap talk will nonetheless achieve efficiency. The conjecture is tested with one-way communication. When the sender first chooses a signal and then an action, there is impressive coordination: a 94\% probability for the potentially efficient (but risky) play, given a signal for efficient play. Without communication, efforts to achieve efficiency were unsuccessful, as the proportion of B moves is only 35\%. I also test a hypothesis that the order of the action and the signal affects the results, finding that the decision order is indeed important. While Aumann's conjecture is behaviorally disconfirmed when the signal is determined initially, the signal's credibility seems to be much more suspect when the sender is known to have first chosen an action, and the results are not statistically distinguishable from those when there is no signal. Some applications and issues in communication and coordination are discussed.
\end{abstract}

\section{INTRODUCTION}

Most economists would agree that communication can affect outcomes, particularly in environments with multiple equilibria. The possibilities inherent from costly signaling have been well-explored in the 25 years since Spence (1974). Yet the effectiveness of

\footnotetext{
* I wish to thank Jordi Brandts, Antonio Cabrales, Rosemarie Nagel, and Matthew Rabin for valuable suggestions and ideas. All errors are my own. The financial support of Spain's Ministry of Education under grant D101-7715 is gratefully acknowledged.
} 
costless, non-binding pre-play communication in achieving efficient outcomes has long been a subject of controversy. Since many economic interactions include such "cheap talk," this issue has considerable importance for efficiency and social welfare. ${ }^{1}$ Cheap talk should be effective in achieving coordination in some types of games, but not in others where interests conflict completely or where a signal pooling problem critically impairs the credibility of a message.

Coordination games are of particular interest, as they model the economic contexts where the optimal action of an agent depends on the choice(s) of others and feature multiple equilibria. Often there is an efficient outcome which can only be achieved through coordination; some type of communication is possible, but there is no binding enforcement mechanism available. Generally, we can rank the Nash equilibria on the basis of Paretoefficiency or payoff dominance, so that we can determine the efficient equilibrium and observe the frequency of its realization. Coordination failures are theoretically possible, particularly when there is a risk-dominant equilibrium (Harsanyi and Selten, 1988) which differs from the Pareto-efficient one. The experimental literature suggests that the extent to which communication can enhance coordination may well vary across different forms of games and message technologies.

Aumann (1990) expresses the view that communication cannot affect the outcome of the game if the signaler(s) has/have a strict preference over the other player's strategy choice. He illustrates this view with the following game:

$\begin{array}{ccc} & \text { A } & \text { B } \\ \text { A } & 7,7 & 8,0 \\ \text { B } & 0,8 & 9,9\end{array}$

Both $[\mathrm{A}, \mathrm{A}]$ and $[\mathrm{B}, \mathrm{B}]$ are pure-strategy Nash equilibria, with $[\mathrm{B}, \mathrm{B}]$ payoff-dominant and [A,A] risk-dominant. Aumann points out that it is in a player's interest to always signal B,

1Farrell and Rabin (1996) argue that these situations are more common than those where costly signaling is relevant. 
regardless of whatever strategy she actually intends to use, since each strictly prefers that the other play B. Let us call such a game an Aumann game. He concludes: "Therefore an agreement to play ...[B,B]... conveys no information about what the players will do, and cannot be considered self-enforcing."

The logic is impeccable, but some feel that an expectation that communication will not affect results is unrealistic. Farrell and Rabin (1996) state: "although we see the force of Aumann's argument, we suspect that cheap talk will ... [achieve B,B]." Blume (1998) makes specific reference to Aumann (1990) and finds that effective one-sided communication can be a stable outcome if the risk of the preferred equilibrium is low relative to some standard of comparison. Farrell (1988) states: "it is a matter of whether [a player] decides on his move ... after he chooses his message, or ... his move first and then his message. If the latter, then Aumann's criticism is compelling; if the former, then matters are rather unclear."

Previous experimental work on the results of pre-play communication shows mixed results. Isaac and Walker (1988) find that communication is very effective in a public goods experiment. Van Huyck et al (1990) and Cooper et al (1990) both observe coordination failures. Cooper et al (1992) test a game slightly different than that of the Aumann conjecture and achieve very good coordination with two-way simultaneous signals, but only partial success with one-way signals. Clark et al (1997) do test an Aumann game, using twoway simultaneous signals. They find that messages induce only a relatively low level of coordination, although this level is considerably higher than when there is no signal.

This paper reports the results of a test of Aumann's conjecture, using one-way written signals marked on message forms. Three different Aumann game calibrations are tested, with varying degrees of risk-dominance present. When the sender's signal precedes her action choice (SA), the receiver chooses the potentially efficient action 297 of 315 times (94\%) after she receives a message signaling this action. Moreover, when the sender signals B (315 of 330 signals), the sender also plays B 94\% of the time (296/315). B actions are $91 \%$ of all action choices. Overall, [B,B] is achieved in 284 of 330 cases $(86 \%) ; 90 \%$ 
(282/315) contingent on a B signal. These percentages were surprisingly consistent across the degree of risk-dominance, in contrast to what would be predicted by Blume (1998).

Two types of control sessions were also conducted. First, the games were played without communication. A substantial segment of the population attempts to attain efficiency, at least initially. However, despite an apparent general subject-pool affinity for payoff-dominance, risk-dominance generally prevails here. The $[\mathrm{B}, \mathrm{B}]$ outcome occurred in 64 cases of $330(19 \%)$, while B plays were $35 \%$ of the total. The second control was a prisoners' dilemma with one-way communication. This was used to test whether the written character of the signal was responsible for the high degree of cooperation, due to either a perceived implicit contractual relationship or a "taste for truth-telling." The results are quite different than in the Stag Hunt - even though the cooperative move is signaled $80 \%$ of the time, defection was $90 \%$ of the action choices, by both the sender and the receiver.

As there is currently no experimental evidence for the case when an action is known to precede the message, and since this order reversal would seem to highlight an important cognitive issue, the Farrell (1988) comment is also tested. In this treatment (AS), it is common knowledge that it is physically necessary for a sender to choose her action prior to deciding on a signal. While the payoffs remain unchanged, the participants' perceptions of the cognitive environment may well influence the credibility of the potentially misleading and self-serving message. This environment appears to be characterized by a high degree of uncertainty. The large variation in behavior across groups is similar to, but greater than, the variance observed in sessions where no signal was permitted. The aggregated probability that B is played when a B signal is made is .52. Statistical tests show that the order makes a significant difference in outcomes. However, results for the reversed order treatment and the no signal treatment are not statistically distinguishable. Perhaps this decision order shifts the mean of sextuplets" "signal credibility distribution" to just below the critical level, so that some groups can achieve coordination with the signal, while many others cannot. 
Since the results in the control sessions are so different from those in the SA games, it is difficult not to conclude that the signal is the mechanism by which coordination is achieved. Aumann's argument certainly holds if one believes the probability of a B move conditioned on a B signal to be low. Although the observed level of coordination may not be found in all possible groups, here it seems clear that a B signal does convey information, so that the conjecture is behaviorally disconfirmed when a sender's message precedes her choice of actions. On the other hand, when the sender's action choice is made first, a B signal only achieves the $[\mathrm{B}, \mathrm{B}]$ outcome with probability $46 \%$ and the results are not statistically distinguishable from the no signal case. Behavior is much more consistent with the conjecture when the cognitive issue is highlighted.

In section 2 of this paper, I mention some theoretical issues pertaining to coordination and communication, as well as relevant experimental results. Section 3 describes the experimental design and the results are presented in section 4. Section 5 offers some discussion of the results and issues affecting beliefs about conditional play. Section 6 concludes and presents extensions to "hierarchies" of cheap talk and potential classifications of types of games by the degree of conflict or coordination.

\section{COORDINATION, COMMUNICATION, AND DOMINANCE}

When players' interests are well-aligned, communication can readily achieve coordination. As an example, consider the Battle of the Sexes:

$\begin{array}{ccc} & \text { B } & \text { F } \\ \text { B } & 2,1 & 0,0 \\ \text { F } & 0,0 & 1,2\end{array}$

With a one-way signal, it should be easy to achieve $[\mathrm{B}, \mathrm{B}]$ or $[\mathrm{F}, \mathrm{F}]$ (the outcome depends on who is chosen to signal). Here there is no reason to attempt to deceive the other player. 
The message is self-signaling, as the signaler wishes to convey information if and only if it is true. However, note that coordination is much more problematic with two-way simultaneous messages.

Farrell and Rabin (1996) define a message to be self-committing if it creates incentives for the signaler to fulfill it, if the other player considers the message credible. A credible message must be self-committing. Yet, self-committing messages need not be entirely credible or, as in Aumann (1990), self-enforcing. In the background, there is the issue of payoff-dominance versus risk-dominance. Harsanyi and Selten (1988) find both to be quite relevant in equilibrium selection. On the other hand, Harsanyi (1993) states that further reflection on the Aumann (1990) arguments "have convinced me to ... use only risk dominance as choice criterion among different equilibria without any use of payoffdominance." So the issue of whether payoff-dominance is relevant still seems to be unsettled.

One cannot conclude that simply because a message could be self-serving, it truly is. The behavioral question (on which a payoff-maximizing player should base a choice of actions) is what actions people actually take, given a particular signal. It is easy to calculate the minimum probability assessment required for a neo-classical agent to play $\mathrm{B}$ in response to a B signal. ${ }^{2}$ In a sense, it is this subjective probability estimate that reflects the degree of perceived risk. Even if we neglect any non-pecuniary "warm glow" from achieving efficiency, there is another issue: if the sender assigns a high probability that his signal will be considered to be credible, he does best by playing $\mathrm{B}$. Is it reasonable to believe that the receiver considers the message sufficiently credible? Perhaps so. This may be related to the extent of the influence of payoff-dominance in the population.

2 The critical probabilities are $2 / 3,5 / 6$, and $7 / 8$ for B play in the three calibrations tested. The mixed strategy equilibrium occurs when both players use mix their plays with these critical probabilities. 
Evidence from experimental tests of coordination in games is rather mixed. Van Huyck, Battalio, and Beil (1990) find convergence to the payoff dominant outcome with small fixed groups of subjects, but coordination failure with large groups. Cooper, DeJong, Forsythe, and Ross (1990) observe that forward induction has limited success in generating coordination. Here risk-dominance seems to be the driving force. There are few tests of the effectiveness of pre-play communication with respect to coordination. One game (SCG) tested in the well-known Cooper et al (1992) paper is:

A $\quad$ B

$\begin{array}{ccc}\text { A } & 800,800 & 800,0 \\ \text { B } & 0,800 & 1000,1000\end{array}$

Three conditions are tested: no signals, one-way signals, and simultaneous two-way signals. They report that the efficient outcome is reached, respectively, in $0 \%, 53 \%$, and $90 \%$ of the cases. Although Cooper et al conclude only that two-way communication is effective in achieving efficiency, a one-way signal also has a significant and substantial effect on coordination. However, their payoffs do not permit a direct test of the Aumann conjecture, as there is no strict preference over the other player's action when she intends to play A.

Clark, Kay, and Sefton (1997) does examine the Aumann conjecture directly. ${ }^{3}$ One game used is:

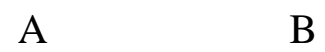
A 700,700
900,0
B $\quad 0,900 \quad 1000,1000$

${ }^{3}$ I became aware of this paper only after conducting my experimental sessions. 
Clark et al find that the likelihood that B is played is $42 \%$, even though B is signaled $81 \%$ of the time, in contrast with the Cooper et al (1992) two-way signal results and their own replication of these SCG results. ${ }^{4}$ However, the corresponding percentage of B play in the game without signals is $19 \%$ and they state (pg. 11) that "the proportion of [B] choices ... is affected by communication ( $\mathrm{p}=.0027)$." Nonetheless, the efficient outcome is not observed in most cases and they conclude on pg. 14 that "these results suggest that communication is not sufficient for attaining efficiency in simple coordination games."

In these tests, the risk-dominant equilibrium is generally observed absent communication, while the payoff-dominant equilibrium is far more prevalent with communication. ${ }^{5}$ Even though the degree of effectiveness of communication varies, signals seem to always significantly increase the likelihood of efficient play in coordination games. But there has been only limited success for one-way signals in experimental $2 \times 2$ coordination games, even though there is some theoretical basis for expecting coordination.

\section{EXPERIMENTAL DESIGN}

This experiment was conducted at Universitat Pompeu Fabra in Barcelona. A total of 162 people participated, with each present at exactly one session. Subjects were recruited by posting notices and were almost exclusively economics, business, and humanities undergraduate students. Average earnings were about \$10, including a show-up fee.

${ }^{4}$ Clark et al find that, in the SCG, subjects played B in $77 \%$ of all cases, while the probability of a B signal was $85 \%$. Overall in their Aumann game, the proportion of B choices conditional on a $[\mathrm{B}, \mathrm{B}]$ announcement was only $50 \%$, compared to $96 \%$ in the replication of Cooper et al.

5 Naturally, there is also a mixed-strategy equilibrium. 
There were generally 12 or 18 people in each session. ${ }^{6}$ Subjects were seated at carrels separated by dividers and a ban on verbal communication was enforced. Instructions were given to each person and were also read aloud to ensure a common knowledge condition. There were 10 periods in each session. Participants were told that there would be random re-matching in each period and that the role of the signaler was also randomly drawn for each period. While there was indeed random matching in each period, each group was actually subdivided into groups of 6 . The point of this design is to obtain independent data points, since each group's actions are internally interdependent. With separate groups of 6 , we can treat each cell as a single independent data point. ${ }^{7}$ While it is true that two people were sometimes paired more than once, no player was ever aware of the identity of the other player in the pair. As anonymity was ensured and there were generally 18 participants in the session, subjects had little reason to be concerned with repeat game effects. English translations of the instructions are presented in Appendices A and B (payoffs are sample payoffs and differ across the games). Prior to each period, every participant was given a piece of paper. Senders received a paper which read "I intend to play [A or B]." 8 The signal form is shown in Appendix C. For receivers, the initial paper received was blank. Identical black pens were provided to each person, with which the signal was indicated on the paper. In this way, no one knew which people made signals in a round. After signals were marked, papers were collected and sorted; signals were distributed to assigned receivers and senders received blank pieces of paper. At this point, players marked play choices on a decision form, a sample of which is also shown in Appendix C. These forms were collected and the payoffs were determined and marked on

\footnotetext{
6A few sessions had only 6.

${ }^{7}$ This is a consistent point of the Bonn school. I am indebted to Rosemarie Nagel for this suggestion.

${ }^{8}$ This signal was in Catalan, courtesy of Andreu Mas-Colell. The corresponding signal in the AS treatment was "I indicate that my play is [A or B]" in Catalan and was provided by Jordi Brandts.
} 
the forms, which were then returned to the participants. Payoffs were aggregated over the 10 periods in the session and subjects were paid individually and privately.

Three different payoff calibrations were used in the coordination game, to investigate the degree of sensitivity to risk-dominance. These, along with the Prisoner's Dilemma played, are shown in Figure 1.

Stag Hunt

A
A $70,70 \quad 80,50$
B $50,80 \quad 90,90$

(2)
A 70,70

A

B

B 10,80
B

A $70,70 \quad 80,0$ A $70,70 \quad 120,10$

90,90

Prisoner's Dilemma

(3)

A B
A B 90,90

In the AS treatment (Appendix D), games 1-3 were played. Senders completed their decision sheets before receiving message forms; as in the other signal treatments, after these message forms were completed and distributed, the receivers made their decisions.

\section{RESULTS}

\section{Signal followed by action (SA)}

The results are striking. In the Stag Hunt variations, the proportion of B signals was 95\%. Conditional on a B signal, B was played by both the sender and the receiver with $94 \%$ probability and the efficient outcome achieved in 282 of 315 cases (90\%). Overall, the

proportion of B plays was $91 \%$ and the likelihood of the efficient outcome was $86 \%$. Aggregated results are presented in the Cooper et al (1992) format in Table 1. Details for each 6 person cell can be found in Appendix D.

Table 1 - Signal, then action 
Announcement

A

B

Total

Announcement

A

B

Total

Announcement

A

B

Total

Announcement

A

B

Total

Game (1)

Signaler, Receiver play

$\begin{array}{ccccc}\text { A,A } & \text { B,B } & \text { A,B } & \text { B,A } & \text { Total } \\ 4 & 1 & 2 & 1 & 8 \\ 1 & 100 & 4 & 7 & 112 \\ 5 & 101 & 6 & 8 & 120\end{array}$

\section{Game (2)}

Signaler, Receiver play

$\begin{array}{ccccc}\text { A,A } & \text { B,B } & \text { A,B } & \text { B,A } & \text { Total } \\ 2 & 1 & 2 & 1 & 6 \\ 1 & 105 & 3 & 5 & 114 \\ 3 & 106 & 5 & 6 & 120\end{array}$

\section{Game (3)}

Signaler, Receiver play

$\begin{array}{cc}\text { A,A } & \text { B,B } \\ 1 & 0 \\ 2 & 77 \\ 3 & 77\end{array}$

A,B

0

8

8

Total - Games (1),

$\begin{array}{cc}\text { B,A } & \text { Total } \\ 0 & 1 \\ 2 & 89 \\ 2 & 90\end{array}$

Signaler, Receiver play

$\begin{array}{ccccc}\text { A,A } & \text { B,B } & \text { A,B } & \text { B,A } & \text { Total } \\ 7 & 2 & 4 & 2 & 15 \\ 4 & 282 & 15 & 14 & 315 \\ 11 & 284 & 19 & 16 & 330\end{array}$

The results for these three calibrations are remarkably similar. There is no appreciable difference for either the proportion of B plays (.90, .92, and .91 for Games 1, 2, and 3, respectively) or the proportion of $[\mathrm{B}, \mathrm{B}]$ outcomes $(.84, .88$, and .86$)$. A Kruskal-Wallis test, using each sextuple as one observation, fails to reject the hypothesis that the behavior is the same across calibrations $\left(\chi^{2}=.268\right.$, d.f. $=2$, n.s. $)$.

An examination of the behavior of the receivers (Appendix E) shows that the vast majority of subjects considered a B signal to be credible. $83 \%$ of subjects always responded 
to a B signal by playing B. Overall, 61 of 66 receivers (92\%) played B at least $75 \%$ of the time when a B signal was received . Given the senders' behavior in every sextuple, a B play was payoff-maximizing ex post for a receiver and thus it was also optimal for a sender to signal B and to then play B.

\section{No signals (NS)}

In order to test the effect of the signal, it is necessary to compare these results with the play in the sessions without communication. The aggregated data for the no communication treatments is presented in Table 2 .

\section{Table 2 - No signal}

$\begin{array}{cccccc}\text { Game } & \text { B plays } & \text { A plays } & {[\mathrm{B}, \mathrm{B}]} & \text { Off-diagonal } & {[\mathrm{A}, \mathrm{A}]} \\ (1) & 40 \% & 60 \% & 21 \% & 39 \% & 40 \% \\ (2) & 35 \% & 65 \% & 13 \% & 44 \% & 43 \% \\ (3) & 29 \% & 71 \% & 17 \% & 23 \% & 60 \%\end{array}$

There is a trend toward more risk-dominant (A) plays with a higher degree of riskdominance. However, even if we make the rather dubious assumption that every decision is independent, only the difference between the proportions of B plays in games 1 and 3 are significant at conventional levels. ${ }^{9} \quad$ A Kruskal-Wallis test, using each sextuple as one observation, fails to reject the hypothesis that behavior is the same in all three games $\left(\chi^{2}=\right.$ .825 , d.f. $=2$, n.s.). There is considerable variation across sextuples, as can be seen in Appendix D. Some groups begin with very high proportions of B moves and maintain substantial coordination, while others start with few B moves and rapidly converge to all A plays. It was ex post payoff-maximizing to play A in all of the no communication sessions.

${ }^{9}$ For the comparison between games 1 and 3 , we have $\chi^{2}=7.22, \mathrm{p}<.01$; for games 1 and 2 , $\chi^{2}=1.81$ (n.s), and for games 2 and $3, \chi^{2}=2.56$ (n.s). 
A conservative nonparametric statistical test, the Wilcoxon-Mann Whitney test on individual sextuples, shows that there is a strongly significant difference $(\mathrm{p}<.0001)$ between the SA and no communication treatments for both the proportion of B moves and the proportion of $[\mathrm{B}, \mathrm{B}]$ outcomes. It is obvious that the signal has a strong behavioral effect on play, even though theory correctly points out that a B signal is potentially a mixed message.

There are also trends for the proportion of B plays across time. This proportion decreases steadily and substantially without communication, while with a signal it is always high, but increases slightly from initial levels before a mild decay at the end (Figure 2). Although 10 periods may not be enough to achieve equilibrium, the $[\mathrm{A}, \mathrm{A}]$ equilibrium is increasingly likely over time absent communication and the $[\mathrm{B}, \mathrm{B}]$ equilibrium seems fairly stable. ${ }^{10}$ An additional benefit is that the signal helps avoid the highly inefficient off-diagonal outcomes (Figure 5).

\section{Prisoner's Dilemma (PD)}

As the proportion of B play is so high compared to some other results and a written signal was used rather than a computer message, a Prisoner's Dilemma game control was conducted as a control to investigate whether signalers played B simply because they felt "contractually bound" by their marked signals. This data is presented below:

\section{Table 3 - Prisoner's Dilemma}

Announcement

A

B

Total
$\mathrm{A}, \mathrm{A}$

15

59

74
Signaler, Receiver play

$\begin{array}{cccc}\text { B,B } & \text { A,B } & \text { B,A } & \text { Total } \\ 1 & 2 & 1 & 19 \\ 1 & 5 & 6 & 71 \\ 2 & 7 & 7 & 90\end{array}$

${ }^{10}$ The relative stability of B play is evidence against the view that there is some ineluctable reputation effect or a public good provision explanation for the results. 
Here there were only $10 \%$ B plays overall, despite the fact that the proportion of B signals was $79 \%$. The probability of a B play conditioned on a B signal was only $12 \%$ for the signaler and $10 \%$ for the receiver. Senders clearly did not feel bound to comply with their marked signals and receivers did not view these signals as credible. While the urge towards payoff-dominance may be high in this subject pool, this influence can be overcome by clear disincentives.

The PD results show that a written signal is not sufficient to achieve coordination in all games. We see that subjects are willing to misrepresent their intentions when it suits them. However, one cannot conclude from this that a written signal in the SA treatment does not lead to different behavior than would a computer signal. It may well be true that there is a greater aversion to misrepresentation with a written signal than otherwise. Any such degree of aversion must be considered, along with the underlying environment and payoff structure, when assessing the credibility of a signal.

\section{Action followed by signal (AS)}

When it is common knowledge that senders have already chosen their actions, some subjects appear to focus more on the self-interest problem noted by Aumann. Results were highly group-specific. For the 6 groups, two featured nearly all-B play, 2 quickly became nearly all-A, and the other 2 were still mixed after 10 periods. Thus, in some cases the urge toward payoff-dominance is strong enough to induce and sustain B plays. In others, cooperation breaks down rapidly and completely. Results for each sextuple are given in Appendix D, while a summary is presented below:

\section{Table 4 - Action, then signal}

Announcement Signaler, Receiver play
A,A
B,B
A,B
B,A
Total 


$\begin{array}{cccccc}\text { A } & 32 & 0 & 0 & 1 & 33 \\ \text { B } & 48 & 68 & 15 & 16 & 147 \\ \text { Total } & 80 & 68 & 15 & 17 & 180\end{array}$

B signals were still the norm (82\%), but the overall probability of a B play, given a B signal $(\mathrm{p}[\mathrm{B} \mid \mathrm{s}(\mathrm{B})])$, is only .52. However, the variance is quite high and the probability by sextuple ranges from a low of .07 (3 of 46) to a high of 1.00 (60 of 60).

The Wilcoxon test allows us to test whether the results for these sextuples differ significantly from those for groups in other treatments. Comparing the (AS) results with data from the SA treatment, we can reject the hypothesis that there is no difference in treatments at $\mathrm{p}=.03(\mathrm{z}=1.96) .{ }^{11}$ On the other hand, this test cannot reject the corresponding hypothesis for the NS/AS comparison $(\mathrm{z}=0.33, \mathrm{n} . \mathrm{s})$.

\section{Summary}

The degree of coordination with a one-way signal is quite high when the signal precedes an action. There is a strong contrast to both control treatments, indicating that this degree is not invariant for the subject pool. Coordination and efficiency are much lower without a signal, so that one can easily reject the hypothesis that the signal conveys no information. The starkly different results in the Prisoner's dilemma show that it is possible for a game's payoff structure to overcome tendencies to adhere to one's signal, so that a written signal cannot per se always be taken at face value. In addition, it seems that the order of the sender's decisions can make a major difference in behavior. Perhaps the reversal of the order of signal and action brings the cognitive task and self-interest issue into sharper focus, reducing the credibility of a signal. The Wilcoxon test shows that there is a significant order effect. Yet this test does not reject (at conventional significance levels) the hypothesis that outcomes are the same for the NS and the reverse order treatments. In this

${ }^{11}$ The data from the three SA calibrations is pooled, given that the Kruskal-Wallis test finds little difference across the games. Similarly, the data from the NS treatment is pooled for the Wilcoxon test against the AS treatment. 
sense, Farrell's comment on Aumann's conjecture is borne out by the data. One might conjecture that while there are some subjects who still seek payoff-dominance, it is not the signal per se that induces any consequent coordination on B plays.

A comparison across all treatments:

\section{Table 5 - Data Summary}

$\begin{array}{ccccccccc}\begin{array}{c}\text { Treatmen } \\ \mathrm{t}\end{array} & \text { B plays } & \text { A plays } & {[\mathrm{B}, \mathrm{B}]} & {[\mathrm{A}, \mathrm{A}]} & \begin{array}{c}\text { Off- } \\ \text { diagonal }\end{array} & \mathrm{p}[\mathrm{s}(\mathrm{B})] & \mathrm{p}[\mathrm{s}(\mathrm{A})] & \mathrm{p}[\mathrm{B} \mid \mathrm{s}(\mathrm{B})] \\ \mathrm{SA} & 91 \% & 9 \% & 86 \% & 11 \% & 3 \% & .955 & .045 & .94 \\ \mathrm{NS} & 35 \% & 65 \% & 16 \% & 47 \% & 37 \% & - & - & - \\ \mathrm{AS} & 47 \% & 53 \% & 38 \% & 44 \% & 18 \% & .817 & .183 & .52 \\ \mathrm{PD} & 10 \% & 90 \% & 2 \% & 82 \% & 16 \% & .789 & .211 & .11\end{array}$

\section{DISCUSSION}

When decisions follow the sequence of one's signal preceding one's action, one-way pre-play communication achieves a high degree of coordination on the efficient outcome in the Stag Hunt. As this is far greater than the degree of coordination without communication (although it seems there are still some attempts to achieve efficiency), the results are different than what would be expected if the signal indeed conveyed no information. On the other hand, in the AS treatment, where the cognitive task seems highlighted by the order reversal, subjects' views concerning the credibility of a B signal differ sharply. Some groups can coordinate on $[\mathrm{B}, \mathrm{B}]$ outcomes, while others coordinate on $[\mathrm{A}, \mathrm{A}]$ outcomes. While the AS results do differ significantly from the SA results, they cannot be statistically distinguished from what is observed when signaling is not possible. The order of decisions is important and, as Farrell hypothesized, the Aumann conjecture is much more behaviorally realistic when signals follow actions. 
Payoff-dominance seems to be a strong influence here, although risk-dominance certainly serves to discipline most players when no communication is possible or when the credibility of a signal is perceived to be low. The Prisoner's Dilemma control shows that risk-dominance is pre-eminent when there are strong disincentives for attempting to reach efficiency, regardless of whether the efficient move has been signaled. We can conclude it is not just a taste for honesty or a perception of an implicit contract that drives the high degree of coordination we observe here in the SA games. Surprisingly, given the Blume (1998) results, varying the degree of risk-dominance has little effect, as the differences in inherent risk in the three calibrations is not reflected in subjects' behavior.

While if one views the SA sequence to be the natural interpretation, the Aumann conjecture is clearly disconfirmed, it is not unreasonable to view the AS sequence as more realistic in some economic environments. Here it seems that anything can happen and we cannot reject Aumann's conjecture. Perhaps this sequence simply helps people to see the subtlety of the problem and as some sophisticated groups might not need this aid, their behavior in the SA treatment would be different than these results. But perhaps not - it is also possible that subjects anticipated that other players would seek to achieve payoffdominance and judged this goal to be feasible.

We can attempt to assess the economic significance of a signal's effectiveness by creating a payoff efficiency index. Absent a signal, each payoff calibration would yield some expected per player payoff if decisions were made randomly and with equal probability. In the Stag Hunt, there is also a clearly defined Pareto-optimal set of payoffs. I define efficiency as $\mathrm{e}=\Pi_{\mathrm{a}} /\left(\Pi_{\mathrm{m}^{-}} \Pi_{\mathrm{r}}\right)$, where the subscripts refer to actual average payoffs, maximum possible payoffs, and expected random-action payoffs, respectively.

\section{Table 6 - Payoff efficiency}

Treatment \# of Average Expected Maximum Efficiency 


$\begin{array}{cccccc} & \text { subjects } & \text { Payoff } & \text { random payoffs } & \text { possible payoffs } & \\ \text { SA1 } & 24 & 86.25 & 72.5 & 90 & .786 \\ \text { SA2 } & 24 & 85.37 & 62.5 & 90 & .832 \\ \text { SA3 } & 18 & 83.78 & 60 & 90 & .793 \\ \text { Avg. SA } & 66 & & & & \mathbf{. 8 0 4} \\ \text { NS1 } & 24 & 72.21 & 72.5 & 90 & -.017 \\ \text { NS2 } & 36 & 59.22 & 62.5 & 90 & -.119 \\ \text { NS3 } & 24 & 66.75 & 60 & 90 & .225 \\ \text { Avg. NS } & 84 & & & & \mathbf{. 0 3 0} \\ \text { AS } & 36 & 72.22 & 62.5 & 90 & \mathbf{. 3 5 4}\end{array}$

Communication induces an $80 \%$ efficiency level in the SA treatment, with an individual obtaining, on average, about $95 \%$ of the maximum possible payoff of 90 . We see that the average payoff achieved in the no signal condition is quite close to that expected with random actions. ${ }^{12}$ Yet the AS efficiency level is substantially better than zero. AS average payoffs are higher when a B signal is made - 73.98 to 69.24 - but both cases are better than the random-action expected payoff of $62.5 .^{13}$ This action, signal sequence still is sufficient to help pairs that might otherwise be uncertain to coordinate on $[\mathrm{A}, \mathrm{A}]$. The degree of coordination on an on-diagonal outcome improves with time, as can be seen in Figure 5. Even though the overall proportion of A and B choices are similar in the NS and AS treatments, the data shows that there are relatively few off-diagonal outcomes in both the AS and SA cases. The AS treatments have fewer off-diagonal outcomes and more $[\mathrm{A}, \mathrm{A}]$ outcomes than the NS treatment. Since 34\% (32/95) of the senders who play A choose to also signal this action and since A signals lead to coordination on $[\mathrm{A}, \mathrm{A}]$ with probability .97 , A signals are one source of the improved AS payoff efficiency. Since it is the off-diagonal cells which are the most "socially costly," any form of coordination is better than none.

${ }^{12}$ The resulting near-zero efficiency level makes the normalization used potentially plausible. ${ }^{13}$ In fact, the likelihood of an $[\mathrm{A}, \mathrm{A}]$ outcome given a $\mathrm{B}$ signal is higher than the probability of an $[A, A]$ outcome without a signal. Table 4 shows that $61 \%(48 / 79)$ of B signals induced [A,A] outcomes, compared to the 55\% (197/360) probability of an [A,A] outcome without a signal. This apparent reversal is in keeping with the nonconsequentalist reasoning discussed in Shafir and Tversky (1992) and Croson (1997). 
The results differ somewhat from those of previous studies, but the combination of message technology and payoff structure also differs. Cooper et al (1992) have limited success with one-way signals and imply that these may be insufficient in many contexts. Yet, if we look at their data from the first 11 periods of their sessions, there was a $65 \%$ chance of a $[\mathrm{B}, \mathrm{B}]$ outcome and a probability of .82 that a receiver played $\mathrm{B}$ in response to a one-way B signal. Absent communication, these periods show a $10 \%$ probability for the $[\mathrm{B}, \mathrm{B}]$ outcome and a $55 \%$ chance for the $[\mathrm{A}, \mathrm{A}]$ outcome, with the proportion of $\mathrm{B}$ moves at $.28 .{ }^{14}$ It would appear that one-way signals do have some beneficial effect in their study. However, the game used is not an Aumann game, as there is no strict preference for the other player's move when one plans to play A.

The contrast with the Clark et al (1997) data is somewhat surprising. Nonetheless, although the Clark et al results are lower in all cases, cheap talk did significantly increase B play and $[\mathrm{B}, \mathrm{B}]$ outcomes in both studies. Various design factors might have induced the differences in observed behavior. First, in an Aumann game, inferences with two-way simultaneous signals may be more complex and the credibility of a $B$ signal thus diminished. ${ }^{15}$ Second, a written signal may be more credible than a computer signal, although the prisoner's dilemma treatment shows that this written form per se is insufficient to achieve Pareto-efficiency. Finally, although asserting heterogeneity across subject pools is suspiciously convenient, a plausible difference in subjects' beliefs about the allure of payoff-dominance for their group could explain much of the contrast in behavior. ${ }^{16}$ Perhaps a replication across subject pools, with identical message structures, would be useful in

${ }^{14}$ The 1992 paper reports only the data from the last 11 periods of their 22 period session. My thanks to Doug DeJong for providing the complete data set.

15 However, Blume (1998) does indicate that multi-sided messages could be effective.

16 Note that B signals were made only $81 \%$ of the time in their Aumann's conjecture test; moreover, in a third Clark et al game where players have a clear incentive to report truthfully, the chance of a B signal is only $51.5 \%$ and the likelihood of B play is only about $29.5 \%$ (this figure provided by Martin Sefton). 
reconciling the differences. Nonetheless, in both experiments pre-play communication with the SA decision order strongly enhances the degree of coordination in an Aumann game. The only available evidence for the AS decision order indicates that a signal is often not considered credible, but that this sequence is fairly successful in achieving coordination on one of the two on-diagonal payoff cells.

\section{CONCLUSION}

Achieving coordination in economic contexts is an important issue. Aumann (1990) conjectures a limitation on the effectiveness of pre-play communication as a coordination mechanism. Yet when his example is tested with a signal is followed by an action, we see that cheap talk permits average payoffs close to the optimum. Yet the results are somewhat different when the order of decisions is known to be reversed. One cannot assume that what we see here is completely general. However, in a more real-world environment there may be more effective message technologies available and there is often a serious issue of reputation. These factors should both serve to enhance coordination. To the extent to which the laboratory abstracts actual behavior, this has direct implications.

There are a number of extensions for this research and a larger research agenda available. For example, one could explore a different message space where outcomes are suggested and then confirmed or countered sequentially. Frey and Bohnet (1995) investigate the role of types of interaction between participants in a Dictator Game where allocators had to physically put pieces of game money in envelopes for the recipient. ${ }^{17}$ They discuss a possible hierarchy of fairness norms related to degrees of communication. It may be that there are different intensities of cheap talk. ${ }^{18}$ There have been some indications that

${ }^{17}$ In a Dictator Game, one subject in a pair unilaterally determines the division of a common endowment.

18 An increasing ranking might be: disembodied computer signals, written messages, visual 
bargaining is more efficient with more personal communication (i.e, Radner and Schotter (1989) and Schotter, Snyder, and Zheng, 1992). With increasing personal contact, it may be perceived as more of a breach of a social norm to violate a technically non-binding agreement. An obvious conjecture is that situations where payoffs are more conflicting require higher "degrees" of cheap talk. One could rank games on the basis of the degree of opposition inherent in the payoff structure. Dawes, McTavish, and Shaklee (1977) find that personal communication produces significantly more public good provision in a dilemma situation, so that even difficult coordination problems may benefit from some form of cheap talk.

In conclusion, one-way communication can be quite effective in achieving coordination, even when there are valid theoretical reasons for doubting the content of a message. Payoff-dominance is a strong element of the equilibrium-selection process in this experiment, although risk-dominance generally prevails if no signal can be made and can also prevail when actions precede signals. Much of the interaction in the world resembles cheap talk, in that implicit agreements are only indirectly enforceable. Further mapping of the terrain of communication and coordination seems a useful social goal.

identification, verbal contact, and face-to-face communication. 


\section{REFERENCES}

Aumann, R. (1990), "Nash-Equilibria are not Self-Enforcing," in Economic Decision Making: Games, Econometrics and Optimisation, J. Gabszewicz, J.-F. Richard, and L. Wolsey, eds., Elsevier

Blume, Andreas (1998), (get current official title), Games and Economic Behavior. (get date and page numbers)

Clark, K., S. Kay, and M. Sefton (1997), "When are Nash Equilibria Self-Enforcing? An Experimental Analysis," Mimeo, University of Manchester (forthcoming, International Journal of Game Theory)

Cooper, R., D. DeJong, R. Forsythe, and T. Ross (1990), "Selection Criteria in Coordination Games: Some Experimental Results," American Economic Review, 80, 218233

Cooper, R., D. DeJong, R. Forsythe, and T. Ross (1992), "Communication in Coordination Games," Quarterly Journal of Economics, 53, 739-771

Croson, R., (1997) "The Disjunction Effect and Nonconsequential Reasoning in Dominant Strategy Games: Extensions and Limitations," mimeo, University of Pennsylvania

Dawes, R., J. McTavish, and H. Shaklee (1977), "Behavior, Communication, and Assumptions about Other People's Behavior in a Commons Dilemma Situation," Journal of Personality and Social Psychology, 35, 1-11 
Farrell, J. and M. Rabin (1996), "Cheap Talk," Journal of Economic Perspectives, 10, 103118

Farrell, J. (1988), "Communication, Coordination, and Nash Equilibrium, Economics Letters, 27, 209-214

Frey, B. and I. Bohnet (1995), "Institutions Affect Fairness: Experimental Investigations," Journal of Institutional and Theoretical Economics, 151/2, 286-303

Harsanyi, J. (1995), "A New Theory of Equilibrium Selection for Games with Complete Information," Games and Economic Behavior, 8, pp. 91-122

Harsanyi, J. and R. Selten (1988), A General Theory of Equilibrium Selection in Games, MIT Press

Radner, R. and A. Schotter (1989), "The Sealed-Bid Mechanism: An Experimental Study," Journal of Economic Theory, 48, 179-220

Schotter, A., B. Snyder, and W. Zheng (1992), "Bargaining through Agents: An Experimental Study," Mimeo, New York University

Shafir, E. and A. Tversky (1992), "Thinking through Uncertainty: Nonconsequential Reasoning and Choice," Cognitive Psychology, 24, 449-474

Spence, A. (1974), Market Signaling: Informational Transfers in Hiring and Related Screening Processes, Harvard University Press 
Van Huyck, J., R. Battalio, and R. Beil (1990), "Tacit Coordination Games, Strategic Uncertainty and Coordination Failure," American Economic Review, 80, 234-248 


\section{APPENDIX A - SAMPLE NO SIGNAL INSTRUCTIONS}

\section{INSTRUCTIONS}

Thank you for participating in this experiment. For your participation today, you will earn some positive amount of money. This amount of money will be determined by the decisions that you make and the way that these decisions interact with the decisions of others.

There will be 10 rounds in this experiment. In each round, you will be paired with a individual randomly drawn from the other people in the room. There is a new random drawing for each round. You will never be informed of the identity of the person with whom you are paired in any round, either during or after the experiment; similarly, no one will know that they are, or have been, paired with you at any point in time.

In each round, you will be asked to choose either action $\mathrm{A}$ or action $\mathrm{B}$. The person with whom you are paired in a round will simultaneously choose action A or action B, without knowing the action you have selected. The payoffs you receive are shown in the game matrix below:

\section{Player Y}

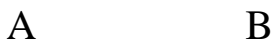
A $\quad 70,70$
80,10

Player X

$$
\text { B } \quad 10,80 \quad 90,90
$$

All payoffs are in pesetas. The first payoff in each pair of payoffs is that of player X; the second payoff is that of player $\mathrm{Y}$. Thus, if both players choose A, each receives 70 pesetas. If player $\mathrm{X}$ chooses $\mathrm{A}$ and player $\mathrm{Y}$ chooses $\mathrm{B}$, then player $\mathrm{X}$ receives 80 and player $\mathrm{Y}$ receives 10. If player $\mathrm{X}$ chooses $\mathrm{B}$ and player $\mathrm{Y}$ chooses $\mathrm{A}$, then player $\mathrm{X}$ receives 10 and player $\mathrm{Y}$ receives 80 . If both players choose $\mathrm{B}$, each receives 90 pesetas. We will go over these possibilities after everyone has read the instructions, to ensure that they are understood by all people present.

At the end of each round, you will be informed of your payoff for that round.

At the end of ten rounds, payoffs for each person will be added up and each person will be paid individually and privately. Please feel free to ask questions before the beginning of this experiment. After the experiment begins, there is to be no communication between the participants in the experiment. Are there any questions? 


\section{APPENDIX B - SAMPLE SIGNAL INSTRUCTIONS}

\section{INSTRUCTIONS}

Thank you for participating in this experiment. For your participation today, you will earn some positive amount of money. This amount of money will be determined by the decisions that you make and the way that these decisions interact with the decisions of others.

There will be 10 rounds in this experiment. In each round, you will be paired with a individual randomly drawn from the other people in the room. There is a new random drawing for each round. You will never be informed of the identity of the person with whom you are paired in any round, either during or after the experiment; similarly, no one will know that they are, or have been, paired with you at any point in time.

In each round, you will be asked to choose either action A or action B. The person with whom you are paired in a round will simultaneously choose action A or action B, without knowing the action you have selected. The payoffs you receive are shown in the game matrix below:

\section{Player Y}

A $\quad$ B
A $\quad 70,70$
80,10

Player X

$$
\text { B } \quad 10,80 \quad 90,90
$$

All payoffs are in pesetas. The first payoff in each pair of payoffs is that of player $\mathrm{X}$; the second payoff is that of player Y. Thus, if both players choose A, each receives 70 pesetas. If player $\mathrm{X}$ chooses $\mathrm{A}$ and player $\mathrm{Y}$ chooses $\mathrm{B}$, then player $\mathrm{X}$ receives 80 and player $\mathrm{Y}$ receives 10. If player $\mathrm{X}$ chooses $\mathrm{B}$ and player $\mathrm{Y}$ chooses $\mathrm{A}$, then player $\mathrm{X}$ receives 10 and player $\mathrm{Y}$ receives 80 . If both players choose $\mathrm{B}$, each receives 90 pesetas. We will go over these possibilities after everyone has read the instructions, to ensure that they are understood by all people present.

Before each round begins, each person will be handed a slip of paper. For half of the people in the room (and the identity of these people will vary from round to round), there will be a blue dot on one side of the paper and a place to check either box A or box B. If you have a slip with a blue dot, please select one of the two boxes. This choice is a statement to the other person in the pair about the intended action for the round to be played. Regardless of which box is checked, the person who checked the box is free to subsequently choose either action in that round. The other half of the people in the round will receive a blank slip. Please do not indicate any choices on the blank slips. 
After a moment or two, someone will come around and collect all of the slips of paper. The slips with blue dots will be distributed to people who were originally given blank slips in that round, according to the assignments which had been randomly drawn. The blank slips will be given to people who were originally given slips with blue dots, according to the assignments which had been randomly drawn. This procedure insures that no other participant in the experiment will know whether or not you were originally given a blue dot. After the exchange of sheets, indicate your decision for the round on your decision sheet.

At the end of each round, you will be informed of your payoff for that round.

At the end of ten rounds, payoffs for each person will be added up and each person will be paid individually and privately. Please feel free to ask questions before the beginning of this experiment. After the experiment begins, there is to be no communication between the participants in the experiment, except for the information on the slips of paper. Are there any questions? 


\section{APPENDIX C - SAMPLE SIGNAL AND DECISION SHEETS}

SA SIGNAL SHEET

\section{INTEND TO PLAY}

A $\quad$ B

AS SIGNAL SHEET

\section{INDICATE MY PLAY IS}
A
B

DECISION SHEET

PLAYER 8

PERIOD 7 


\section{MY DECISION}

MY PAYOFF

A B 


\section{APPENDIX D - RESULTS FOR 6-TUPLES}

Signal 6-tuples

Totals

$\begin{array}{llllllllllll} & & \mathbf{A} & \mathbf{B} & \mathbf{A} & \mathbf{B} & \mathbf{A} & \mathbf{B} & \mathbf{A} & \mathbf{B} & \mathbf{A} & \mathbf{B} \\ \text { SA 1 } & \mathbf{A} & 0 & 0 & 5 & 5 & 0 & 0 & 0 & 1 & 5 & 6 \\ \text { (50's) } & \mathbf{B} & 0 & 30 & 7 & 13 & 0 & 30 & 1 & 28 & 8 & 101 \\ & & & & & & & & & & & \text { Totals } \\ & & & & & & & & & & & \\ & & \mathbf{A} & \mathbf{B} & \mathbf{A} & \mathbf{B} & \mathbf{A} & \mathbf{B} & \mathbf{A} & \mathbf{B} & \mathbf{A} & \mathbf{B} \\ \text { SA 2 } & \mathbf{A} & 0 & 0 & 1 & 1 & 1 & 4 & 1 & 0 & 3 & 5 \\ (10 \text { 's }) & \mathbf{B} & 0 & 30 & 1 & 27 & 5 & 20 & 0 & 29 & 6 & 106\end{array}$

$\begin{array}{llllllllll} & & \text { A } & \text { B } & \text { A } & \text { B } & \text { A } & \text { B } & \text { A } & \text { B } \\ \text { SA 3 } & \text { A } & 3 & 8 & 0 & 0 & 0 & 0 & 3 & 8 \\ (0 \text { 's) } & \text { B } & 2 & 17 & 0 & 30 & 0 & 30 & 2 & 77\end{array}$

$\begin{array}{llllllllll} & & \mathbf{A} & \mathbf{B} & \mathbf{A} & \mathbf{B} & \mathbf{A} & \mathbf{B} & & \\ \mathbf{A S} & \mathbf{A} & 27 & 1 & 27 & 2 & 12 & 5 & & \\ (10 \text { 's) } & \mathbf{B} & 2 & 0 & 1 & 0 & 8 & 5 & & \\ & & & & & & & & & \\ & \mathbf{A} & 1 & 0 & 13 & 7 & 0 & 0 & 80 & 15 \\ & \mathbf{B} & 0 & 29 & 6 & 4 & 0 & 30 & 17 & 68 \\ & & & & & & & & & \\ & & & & & & & & & \\ & & \mathbf{A} & \mathbf{B} & \mathbf{A} & \mathbf{B} & \mathbf{A} & \mathbf{B} & \mathbf{A} & \mathbf{B} \\ \mathbf{P D} & \mathbf{A} & 23 & 3 & 25 & 2 & 26 & 2 & 74 & 7 \\ & \mathbf{B} & 4 & 0 & 2 & 1 & 1 & 1 & 7 & 2\end{array}$

\section{No Signal 6-tuples}

$\begin{array}{lclllllll}\text { Game } 1 & \text { AA } & 5 & 8 & 21 & 14 & & & 48 \\ (50 \text { 's }) & \text { BB } & 8 & 7 & 3 & 7 & & & 25 \\ & \text { Off } & 17 & 15 & 6 & 9 & & & 47 \\ & & & & & & & & \\ \text { Game 2 } & \text { AA } & 19 & 24 & 18 & 4 & 4 & 8 & 77 \\ (10 \text { 's }) & \text { BB } & 1 & 1 & 1 & 10 & 4 & 6 & 23 \\ & \text { Off } & 10 & 5 & 11 & 16 & 22 & 16 & 80\end{array}$




$\begin{array}{cccllll}\text { Game } 3 & \text { AA } & 2 & 15 & 28 & 27 & 72 \\ \text { (0’s) } & \text { BB } & 17 & 4 & 0 & 0 & 21 \\ & \text { Off } & 11 & 11 & 2 & 3 & 27\end{array}$

$\mathrm{SA}=$ a signal was followed by an action; $\mathrm{AS}=$ an action was followed by a signal . 


\section{APPENDIX E - INDIVIDUALS' RESPONSES TO B SIGNALS}

\section{Signal followed by action}

Most receivers (55/66) responded with B to all B signals. Only 5 of 66 failed to respond to a B signal with a play of B at least $75 \%$ of the time.

$\begin{array}{ccr}\text { \% B response \# B responses/ \# B signals received } & \text { \# of receivers } & \\ 100 \% & 7 / 7 & 3 \\ & 6 / 6 & 8 \\ 5 / 5 & 29 \\ 4 / 4 & 11 \\ 3 / 3 & 2 \\ & 2 / 2 & 2 \\ & 4 / 5 & 3 \\ 80 \% & 3 / 4 & 3 \\ 75 \% & 2 / 3 & 1 \\ 67 \% & 3 / 5 & 2 \\ 60 \% & 1 / 4 & 1 \\ 25 \% & 0 / 3 & 1 \\ 0 \% & & \end{array}$

\section{Action followed by a signal}

There was a sharp dichotomy in receiver's behavior. $75 \%$ of subjects (27/36) responded to B signals with either consistent A play or consistent B play. The 9 inconsistent receivers generally (8/9) changed from B to A responses over time.

\% B response \# B responses/ \# B signals received

\# of receivers

$\begin{array}{lll}100 \% & 6 / 6 & 4 \\ & 5 / 5 & 6 \\ & 4 / 4 & 2 \\ & 3 / 3 & 1 \\ & 2 / 2 & 1 \\ 80 \% & 4 / 5 & 2 \\ 67 \% & 2 / 3 & 1 \\ 60 \% & 3 / 5 & 1 \\ 50 \% & 2 / 4 & 1 \\ 40 \% & 2 / 5 & 1 \\ 33 \% & 1 / 3 & 3\end{array}$


$0 / 3$

1

$0 / 2$

$0 / 1$ 
FIGURE 2 - B PLAYS BY PERIOD

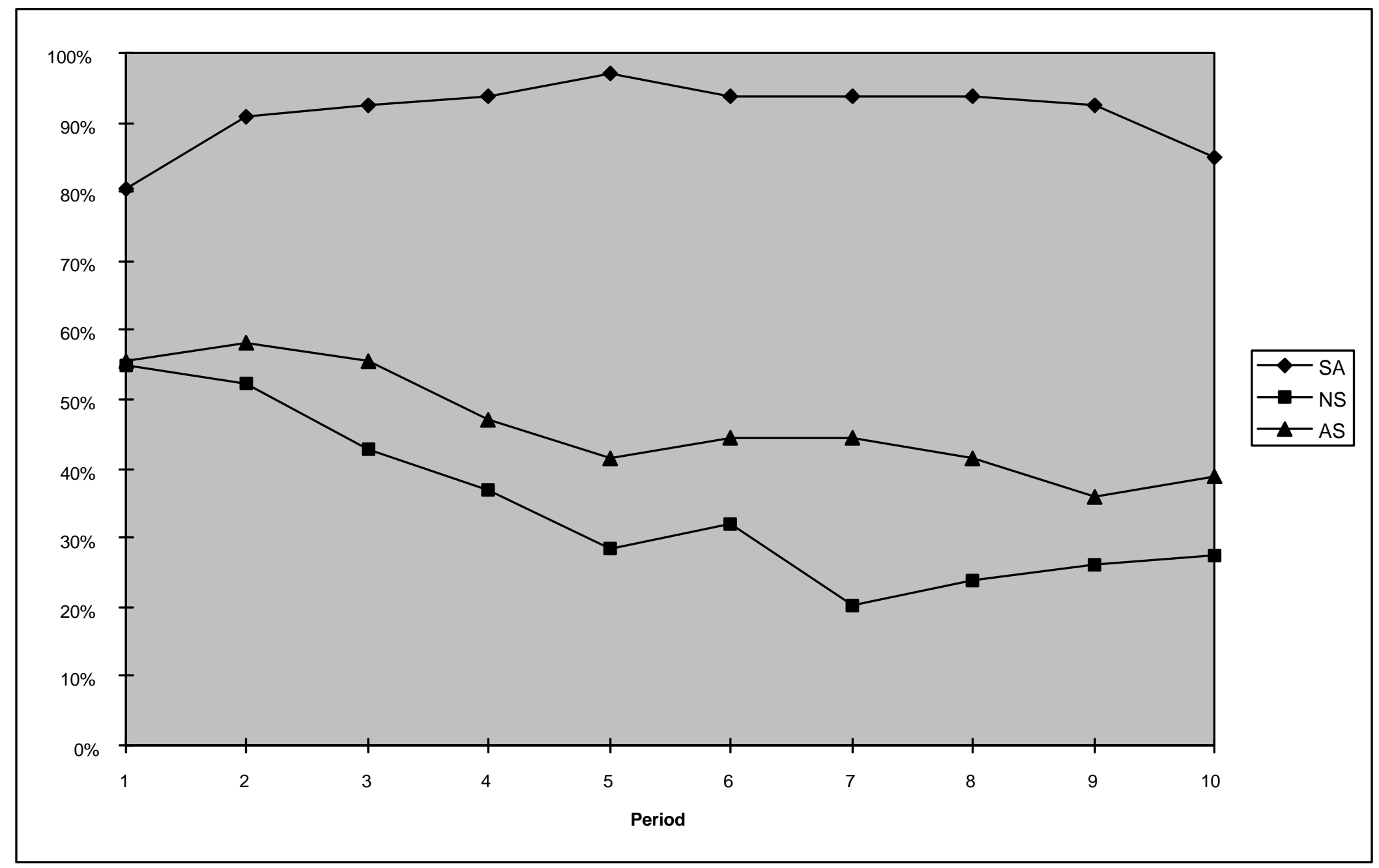


FIGURE 3 - BB RESULTS BY PERIOD

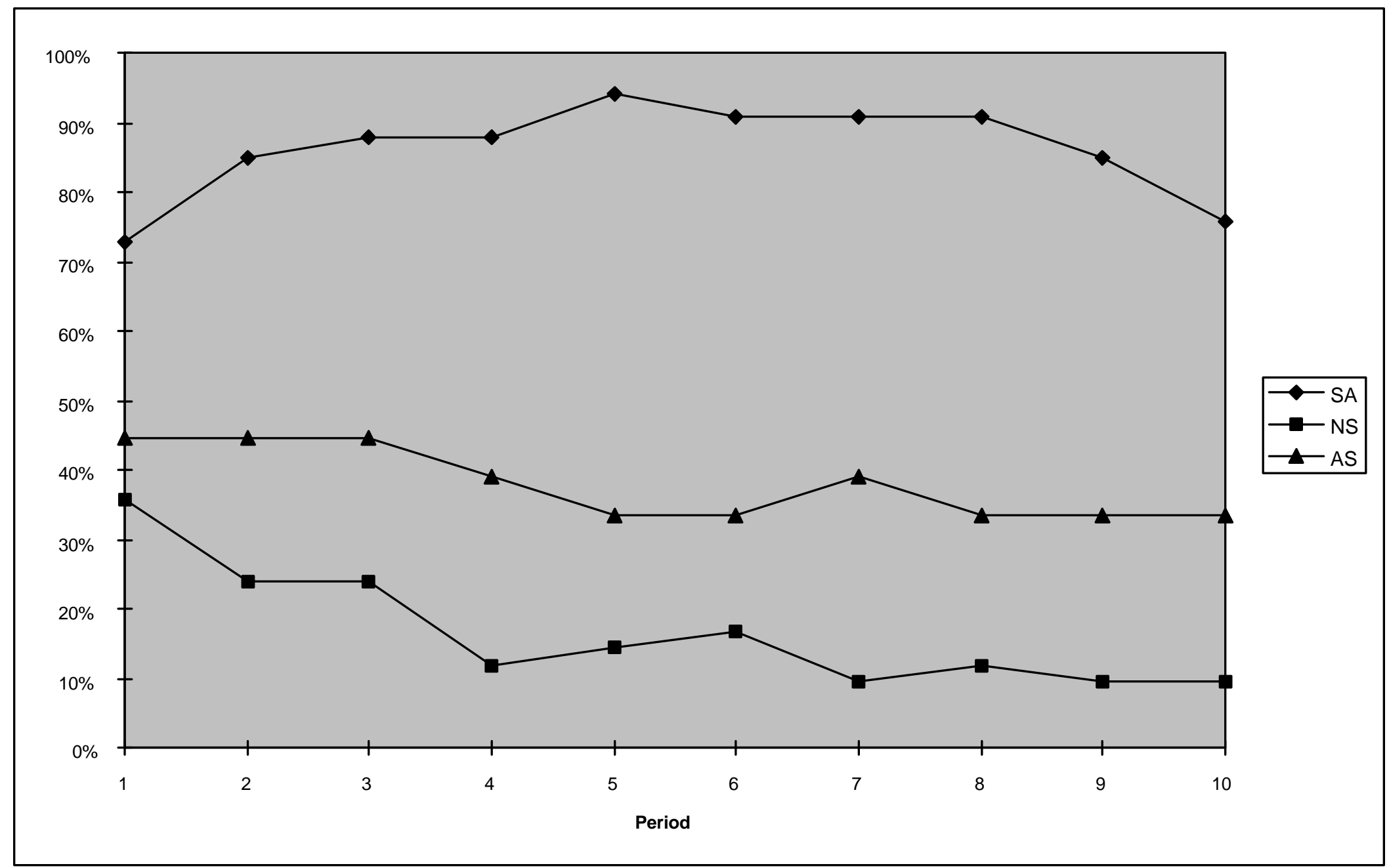


FIGURE 4 - AA RESULTS BY PERIOD

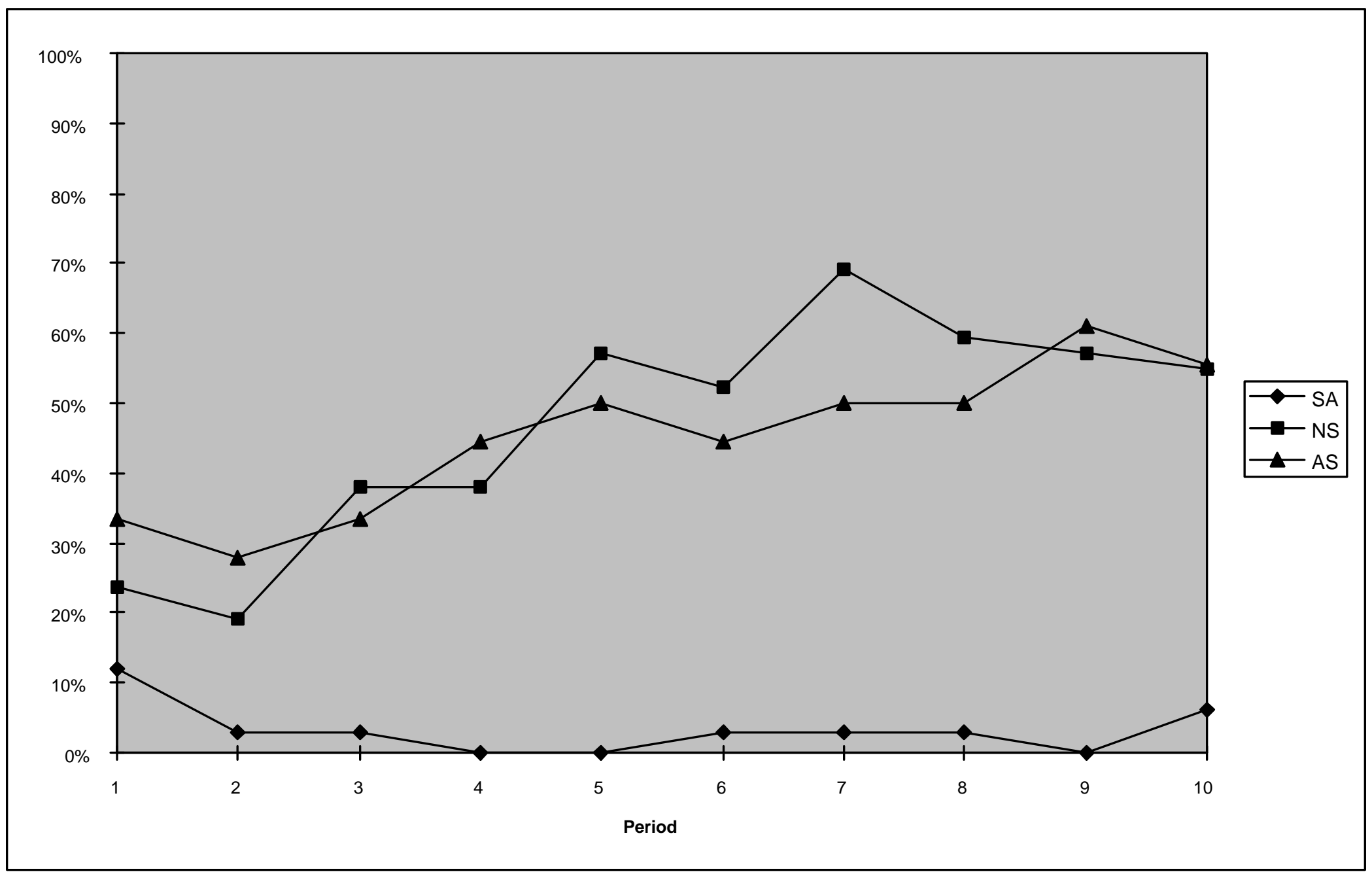


FIGURE 5 - OFF-DIAGONAL RESULTS BY PERIOD

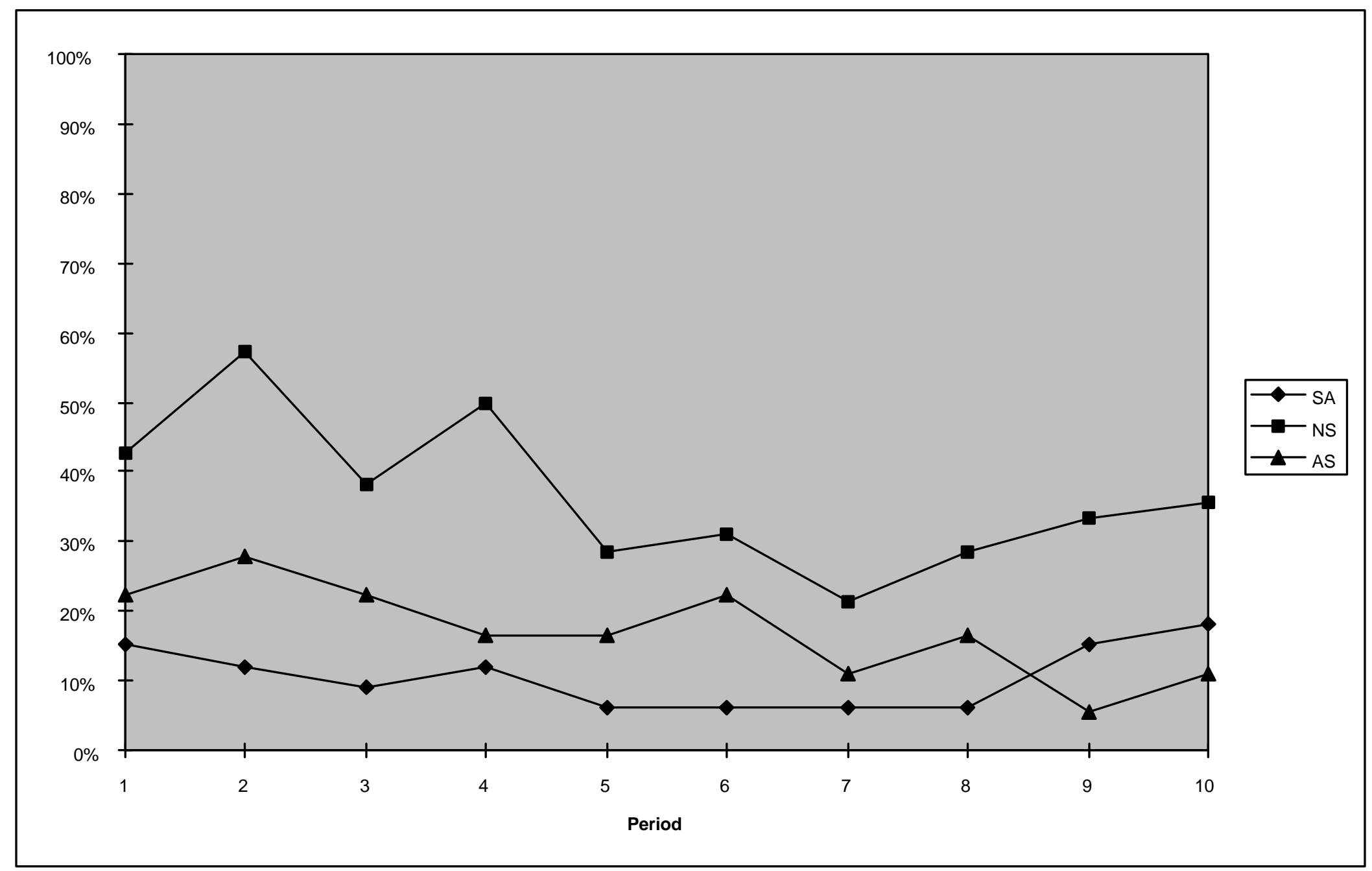


